\title{
Particle acceleration by pulsar wind nebulae inside supernova remnants
}

\author{
Yutaka Ohira*, Shota Kisaka, and Ryo Yamazaki \\ Aoyama Gakuin University \\ E-mail: bhiradphys.aovama.ac.ip
}

\begin{abstract}
The origin of cosmic-ray (CR) PeVatrons is a long standing problem. The CR spectrum breaks at about $1 \mathrm{PeV}$ (so called, the knee energy). The diffusive shock acceleration at supernova remnants (SNRs) is believed to be the acceleration mechanism of CRs up to the knee energy. However, it was estimated that SNRs cannot accelerate CRs to the knee energy without magnetic field amplification. In this study, we propose that CR PeVatrons are pulsar wind nebulae (PWNe) inside SNRs. After a core-collapse supernova explosion, the PWN initially expands into the freely expanding supernova ejecta. Thereafter, the PWN catches up with the reverse shock of the SNR, where particles can be slightly accelerated by the back and forth motion between the PWN and the SNR, and some particles diffuse into the PWN. Afterwards the PWN is compressed by the SNR, where the particles in the PWN are accelerated by the adiabatic compression. Using a Monte Carlo simulation, we show that particles accelerated by the SNR to $0.1 \mathrm{PeV}$ can be reaccelerated to $1 \mathrm{PeV}$ until the end of the PWN compression.
\end{abstract}

35th International Cosmic Ray Conference - ICRC2017

10-20 July, 2017

Bexco, Busan, Korea

\footnotetext{
* Speaker.
} 


\section{Introduction}

The observed cosmic-ray (CR) spectrum can be described by a broken power law. The CR spectrum breaks at about $1 \mathrm{PeV}$ (knee) and about $1 \mathrm{EeV}$ (ankle). It has been thought that the knee energy corresponds to the maximum energy of CR protons accelerated by Galactic supernova remnants (SNRs). Although gamma-ray observations show that $\mathrm{CR}$ protons are accelerated in SNRs [U, []], the proton acceleration to the knee energy has never been observed in SNRs.

The acceleration to the knee energy is also a theoretical problem. For parallel shocks, in order to accelerate protons to the knee energy, magnetic fields must be amplified in the shock upstream and downstream regions. Otherwise the acceleration time scale is too long to accelerate protons to the knee energy. Although many ideas for the magnetic field amplification in the shock upstream region have been proposed, no simulations demonstrate that the upstream magnetic field is sufficiently amplified to accelerate protons to the knee energy. For perpendicular shocks, since particles cannot diffuse to the far upstream region, the acceleration time scale becomes small [B]]. In fact, a recent three-dimensional hybrid simulation showed that particles are injected to diffusive shock acceleration (DSA) at the perpendicular shock in a partially ionized plasma, so that particles are rapidly accelerated by the perpendicular shock [团]. However, for the case of the perpendicular shocks, the maximum energy is not limited by the acceleration time scale but limited by the size of acceleration region [5]. Therefore, the origin of CR knee (so called PeVatrons) is an open issue.

In addition to the knee problem, the origin of heavy CR nuclei is also a long standing problem [6]. The observed Galactic CRs contain more heavy elements than the solar abundance. Since the reverse shock propagates into the metal rich ejecta, the reverse shock of the SNR is thought to be the origin of heavy CR nuclei. However, the magnetic field in the expanding supernova ejecta becomes very small, so that the maximum energy of the accelerated particle at the reverse shock is not so high.

Recently, many young $\gamma$-ray pulsars were discovered by the Fermi Gamma-ray Space Telescope. The number of the observed young $\gamma$-ray pulsars suggests that most core-collapse supernovae leave pulsars with a typical spindown luminosity of $L_{\mathrm{sd}} \sim 3 \times 10^{38} \mathrm{erg} \mathrm{s}^{-1}$ [四]. The pulsars produce a magnetized relativistic wind that makes pulsar wind nebulae ( $\mathrm{PWNe}$ ) expanding into the supernova ejecta. The magnetic field in young PWNe is expected to be strong enough to scatter knee CRs, which is about $B_{\mathrm{PWN}}=0.1-1 \mathrm{mG}$ [ [8, Q Q]. Therefore, PWNe inside SNRs would probably give some contributions to acceleration of knee CRs and heavy CR nuclei.

The magnetic field in the shocked region of SNRs is expected to be strong enough to scatter knee CRs because the downstream super-Alfvénic turbulence amplifies the magnetic field by stretching the magnetic field line. The downstream turbulence is generated by interactions between upstream density fluctuations and the shock front. In addition, the downstream turbulence is generated by the Rayleigh-Taylor instability at the contact discontinuity. Therefore, knee CRs are expected to be confined between the PWN and the shocked region of SNRs. We recently proposed that the PWN-SNR system can accelerate CR to the knee energy [ए]]. In addition, the PWN-SNR system can accelerate heavy CR nuclei to higher energies than a simple SNR system. In this study, using Monte Carlo simulation, we show that the PWN-SNR system actually accelerates particles from $0.1 \mathrm{PeV}$ to $1 \mathrm{PeV}$. 


\section{Time evolution of pulsar wind nebulae inside supernova remnants}

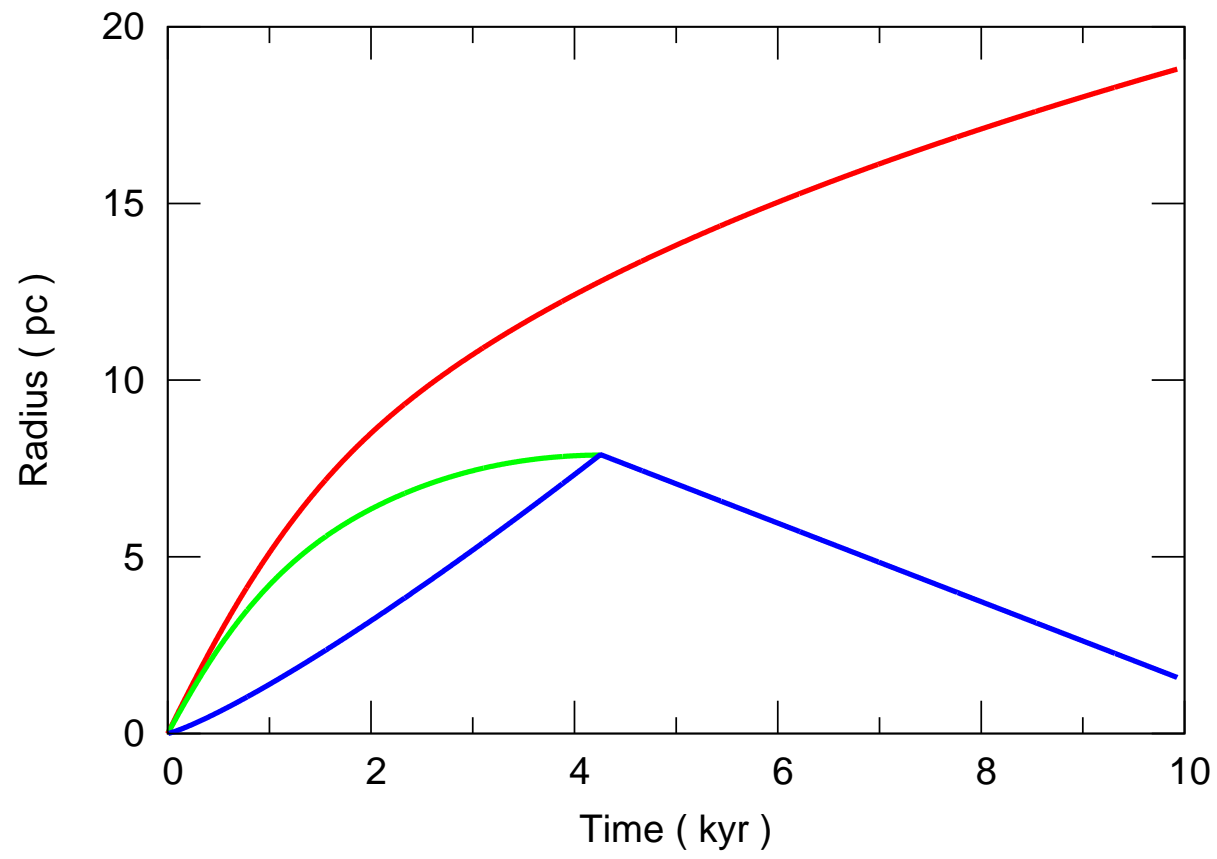

Figure 1: Time evolution of the PWN radius (blue) with a spindown luminosity of $L_{\mathrm{sd}}=10^{38} \mathrm{erg} \mathrm{s}^{-1}$, and radii of the forward (red) and the reverse shocks (green) of the SNR with an explosion energy of $E_{\mathrm{SN}}=$ $10^{51} \mathrm{erg}$, an ejecta mass of $M_{\mathrm{ej}}=6 \mathrm{M}_{\odot}$, and an ambient number density of $n=0.1 \mathrm{~cm}^{-3}$.

In this section, we summarize the time evolutions of the forward and reverse shocks of an SNR. In cases where the initial ejecta distribution and ambient density profiles are uniform, Mckee \& Truelove (1995) [ए]] provided approximate analytical solutions for the forward and reverse shock radii, $R_{\mathrm{SNR}, \mathrm{fs}}$ and $R_{\mathrm{SNR}, \mathrm{rs}}$, of an SNR, that are given by

$$
\begin{aligned}
& R_{\mathrm{SNR}, \mathrm{fs}}=R_{\mathrm{S}} \times \begin{cases}1.37 \frac{t}{t_{\mathrm{S}}}\left\{1+0.60\left(\frac{t}{t_{\mathrm{S}}}\right)^{3 / 2}\right\}^{-2 / 3} & \left(t<t_{\mathrm{S}}\right) \\
\left(1.56 \frac{t}{t_{\mathrm{S}}}-0.56\right)^{2 / 5} & \left(t \geq t_{\mathrm{S}}\right)\end{cases} \\
& R_{\mathrm{SNR}, \mathrm{rs}}=R_{\mathrm{S}} \times \begin{cases}1.24 \frac{t}{t_{\mathrm{S}}}\left\{1+1.13\left(\frac{t}{t_{\mathrm{S}}}\right)^{3 / 2}\right\}^{-2 / 3} & \left(t<t_{\mathrm{S}}\right) \\
\frac{t}{t_{\mathrm{S}}}\left\{0.78-0.03 \frac{t}{t_{\mathrm{S}}}-0.37 \ln \left(\frac{t}{t_{\mathrm{S}}}\right)\right\} & \left(t \geq t_{\mathrm{S}}\right)\end{cases}
\end{aligned}
$$

where $R_{\mathrm{S}}$ and $t_{\mathrm{S}}$ are given by

$$
\begin{aligned}
R_{\mathrm{S}} & \approx 8.73 \mathrm{pc}\left(\frac{M_{\mathrm{ej}}}{6 M_{\odot}}\right)^{1 / 3}\left(\frac{n}{0.1 \mathrm{~cm}^{-3}}\right)^{-1 / 3} \\
t_{\mathrm{S}} & \approx 2 \times 10^{3} \mathrm{yr}\left(\frac{E_{\mathrm{SN}}}{10^{51} \mathrm{erg}}\right)^{-\frac{1}{2}}\left(\frac{M_{\mathrm{ej}}}{6 \mathrm{M}_{\odot}}\right)^{\frac{5}{6}}\left(\frac{n}{0.1 \mathrm{~cm}^{-3}}\right)^{-\frac{1}{3}} .
\end{aligned}
$$

The evolution of an SNR is characterized by the explosion energy, $E_{\mathrm{SN}}$, ejecta mass, $M_{\mathrm{ej}}$, and ambient density, $n$. 
In cases where the spindown luminosity of a pulsar is constant with time and the initial supernova ejecta is uniform, until the PWN interacts with the reverse shock of the SNR, the analytical solution for the PWN radius, $R_{\mathrm{PWN}}$, is given by [12],

$$
R_{\mathrm{PWN}}=1.04 R_{\mathrm{S}}\left(\frac{L_{\mathrm{Sd}} t_{\mathrm{S}}}{E_{\mathrm{SN}}}\right)^{1 / 5}\left(\frac{t}{t_{S}}\right)^{6 / 5}
$$

The spindown luminosity dependence is very weak.

After the PWN interacts with the reverse shock of the SNR, the PWN is compressed by the shocked region of the SNR. This is because the pressure of the shocked region of the SNR is higher than that of the PWN. In this study, we simply assume that the velocity of the PWN during the compression is $v_{\mathrm{PWN}}=-v_{\mathrm{PWN}}\left(t_{\mathrm{c}}\right) / 2$ and the final size of the $\mathrm{PWN}$ is $R_{\mathrm{PWN}}=R_{\mathrm{PWN}}\left(t_{\mathrm{c}}\right) / 5$, where $t_{\mathrm{c}}$ is the time when the PWN reaches the reverse shock of the SNR.

The evolution of the PWN-SNR system is shown in Fig W, where we assume the uniform ejecta $\left(M_{\mathrm{ej}}=6 \mathrm{M}_{\odot}\right)$ and ambient mater profiles $\left(n=0.1 \mathrm{~cm}^{-3}\right)$, and the constant spindown luminosity $\left(L_{\mathrm{sd}}=10^{38} \mathrm{erg} \mathrm{s}^{-1}\right)$. For these parameters, the PWN catches up with the reverse shock of the SNR at $t_{\mathrm{c}} \approx 4 \mathrm{kyr}$. Finally, the PWN size becomes $\approx 2 \mathrm{pc}$ at $t_{\mathrm{end}} \approx 10 \mathrm{kyr}$.

\section{Monte Carlo simulations}

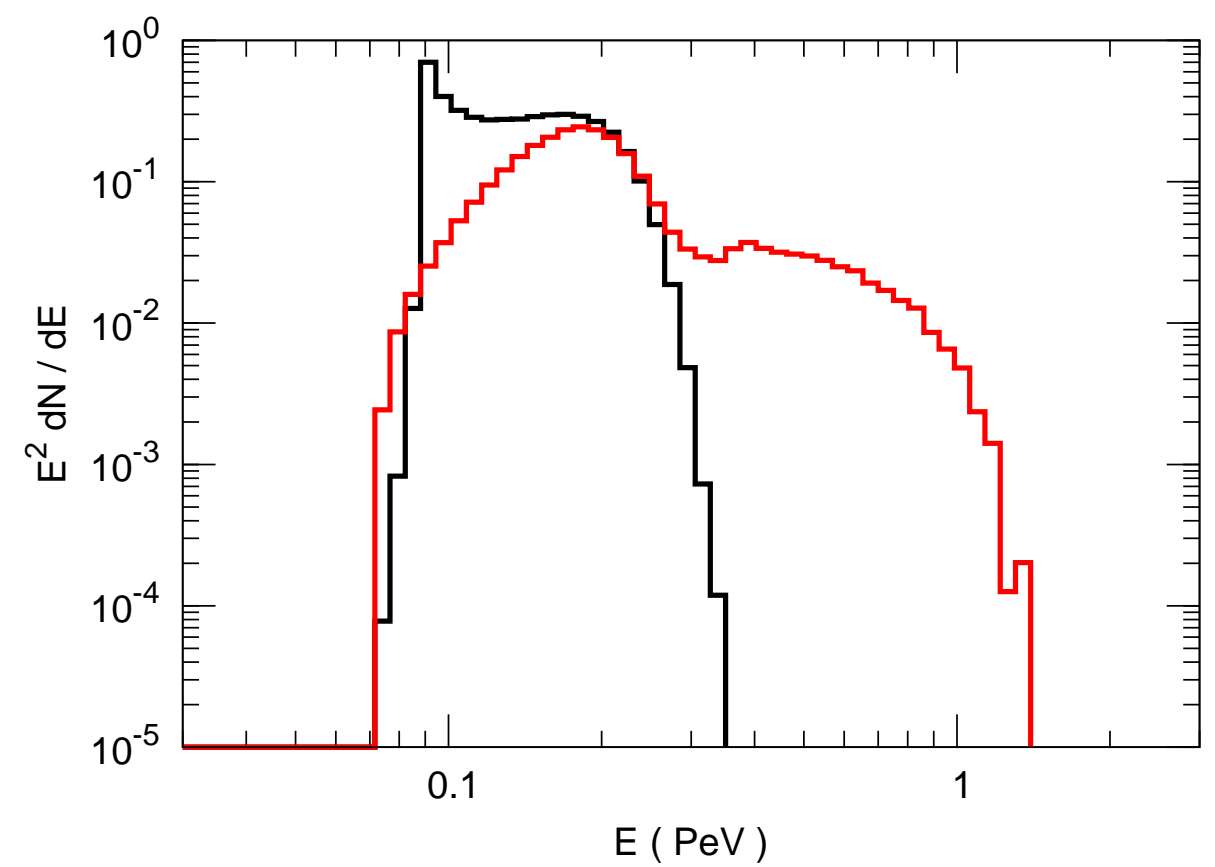

Figure 2: Energy spectrum of reaccelerated particle at the end of the PWN compression, $t=t_{\text {end }}$. The black and red histograms are for particles that were in the SNR $\left(R_{\mathrm{SNR}, \mathrm{rs}}<r<R_{\mathrm{SNR}, \mathrm{fs}}\right)$ and the PWN regions $\left(r \leq R_{\mathrm{PWN}}\right)$ at $t=t_{\mathrm{c}}$ at which the PWN reached the reverse shock of the SNR. The initial energy is $0.1 \mathrm{PeV}$.

To investigate the CR acceleration by the PWN-SNR system, we perform Monte Carlo simulations in this section. We first give the velocity structure in the PWN-SNR system. In the 
reverse shock rest frame, the velocity of the free expanding ejecta is $R_{\mathrm{SNR}, \mathrm{rs}} / t-v_{\mathrm{SNR}, \mathrm{rs}}$, where $v_{\mathrm{SNR}, \mathrm{rs}}=d R_{\mathrm{SNR}, \mathrm{rs}} / d t$ is the propagation velocity of the reverse shock in the observer frame. Then, the downstream flow velocity in the reverse shock rest frame is $\left(R_{\mathrm{SNR}, \mathrm{rs}} / t-v_{\mathrm{SNR}, \mathrm{rs}}\right) / 4$, where the compression ratio at the SNR reverse shock is assumed to be 4 . Hence, the expansion velocity of the shocked ejecta of the SNR in the observer frame is given by

$$
v_{\mathrm{SNR}, \text { shocked }}=\frac{R_{\mathrm{SNR}, \mathrm{rs}} / t+3 v_{\mathrm{SNR}, \mathrm{rs}}}{4},
$$

The expansion velocity of the PWN is given by $v_{\mathrm{PWN}}=d R_{\mathrm{PWN}} / d t$ [12]. In this study, for simplicity, the velocity structures in both the SNR and the PWN are assumed to be uniform until the PWN reaches the reverse shock of the SNR. After the PWN interacts with the reverse shock of the SNR, the PWN is compressed by the shocked region of the SNR. In this study, we assume that the velocity structure in the PWN is given by

$$
\vec{v}_{\mathrm{PWN}, \text { in }}(t, \vec{r})=-\frac{v_{\mathrm{PWN}}\left(t_{\mathrm{c}}\right)}{2} \frac{\vec{r}}{R_{\mathrm{PWN}}(t)},
$$

where $t_{\mathrm{c}}$ is the time when the PWN reaches the reverse shock of the SNR.

Since the shocked region of the SNR and the PWN region can be expected to be highly turbulent, we assume that the $\mathrm{CR}$ motion is a random walk. By using the above hydrodynamical structure, we perform a test-particle Monte Carlo simulation. In this Monte Carlo simulation, simulation particles are isotropically scattered in the local fluid reset frame. The each scattering time is $t_{\mathrm{sc}}=\Omega_{\mathrm{c}}^{-1}\left(E / m_{\mathrm{p}} c^{2}\right)$, where $\Omega_{\mathrm{c}} \approx 10^{-2} \mathrm{~s}^{-1}(B / 1 \mu \mathrm{G})$ is the proton cyclotron frequency, $E$ is the particle energy, and $m_{\mathrm{p}}$ is the proton mass. During the PWN compression phase $\left(t_{\mathrm{c}}<t<t_{\mathrm{end}}\right)$, once the simulation particle escapes from the PWN, we do not follow the particle motion.

In this study, we set the magnetic field to be $B=3 \times 10^{2} \mu \mathrm{G}$ both in the PWN and the shocked region of the SNR, and $B=0$ in the free expanding ejecta. At $t_{\mathrm{inj}}=3 \mathrm{kyr}$, we impulsively inject simulation particles isotropically on the reverse shock sphere, $r=R_{\mathrm{SNR}, \mathrm{rs}}$. Since the forward shock of the SNR can accelerate CRs to about $0.1 \mathrm{PeV}$ [ए]], we set the initial energy to be $0.1 \mathrm{PeV}$.

Fig $\square$ shows the energy spectrum of the accelerated particles at the end of the PWN compression $\left(t=t_{\text {end }}\right)$. The black histogram shows the energy spectrum of particles that were in the shocked region of the SNR when the PWN reached the reverse shock of the SNR $\left(t=t_{\mathrm{c}}\right)$. They are accelerated up to about twice the initial energy by the back and forth motion between the PWN and the shocked region of the SNR. The red histogram shows the energy spectrum of particles confined in the PWN at $t=t_{\mathrm{c}}$. They are further accelerated to $1 \mathrm{PeV}$ by the PWN compression. The energy gain by the PWN compression is given by

$$
\frac{d E}{d t}=-\frac{E}{3} \vec{\nabla} \cdot \vec{v}_{\mathrm{PWN}, \text { in }}=-E \frac{d \log R_{\mathrm{PWN}}}{d t} .
$$

Then, the solution is given by

$$
\frac{E\left(t_{\text {end }}\right)}{E\left(t_{\mathrm{c}}\right)}=\frac{R_{\mathrm{PWN}}\left(t_{\mathrm{c}}\right)}{R_{\mathrm{PWN}}\left(t_{\mathrm{end}}\right)}=5
$$

Since, $E\left(t_{\mathrm{c}}\right) \approx 2 E_{0}$, the particles are finally accelerated to ten times the initial energy, $E_{0}$. We have explored the parameter dependence of acceleration in Ohira et al.(2017) [ए0]. We showed that the energy spectra of accelerated particles do not change significantly as long as $R_{\mathrm{PWN}}\left(t_{\mathrm{c}}\right) / R_{\mathrm{PWN}}\left(t_{\mathrm{end}}\right)=$ 
5 is fixed. This is because dynamics of SNR and PWN do not significantly depend on the spindown luminosity and the ambient number density (see equations ([2.J)-([2.5)). Therefore, if the forward shock of the SNR accelerates protons to $0.1 \mathrm{PeV}$, the PWN-SNR system can reaccelerate the protons to the knee energy.

\section{Discussion and Summary}

In this study, we have investigated the particle acceleration by PWNe inside SNRs. Monte Carlo simulation showed that protons with an energy of $0.1 \mathrm{PeV}$ are accelerated to about $1 \mathrm{PeV}$ as follows. Firstly, the protons are accelerated to $\sim 0.2 \mathrm{PeV}$ by the back and forth motion between the SNR and the PWN. Then, the protons diffuse into the PWN and are accelerated to $\sim 1 \mathrm{PeV}$ by the adiabatic compression while the PWN is compressed by the SNR. Since these processes can be applied to heavy CR nuclei injected at the reverse shock of the SNR and the forward shock of the PWN, the PWN-SNR system is a potential candidate for the origin of heavy CR nuclei.

As one can see in Fig 凤, most particles are accelerated to twice the initial energy. On the other hand, a small fraction are accelerated to $1 \mathrm{PeV}$. We considered only particle diffusion caused by the magnetic field fluctuation, but diffusion by turbulence could be significant. If more particles diffuse deeper inside the PWN by the turbulent diffusion, more particles will be accelerated to $1 \mathrm{PeV}$. In this study, we considered only the impulsive injection of CRs with $E=0.1 \mathrm{PeV}$. In reality, CRs accelerated by the SNR would be continuously injected with an energy spectrum to the PWN. Furthermore, since there is the potential difference of about $1 \mathrm{PV}$ in the PWN, protons could be accelerated to $1 \mathrm{PeV}$ by drifting the toroidal magnetic field [113], [4]], which was not considered in this study. In addition, to understand the source spectrum of CRs that are injected to our Galaxy, we have to consider the spectrum of particles that have escaped from the SNR [15], [6] and variety of the PWN-SNR system. Although we assumed spherical symmetry for the PWN-SNR system in this study, in reality, the PWN-SNR system is more complicated. In particular, the strong turbulence could play an important role, that accelerates particles by turbulent acceleration [प]]. In order to address above problems, we need a more realistic magnetohydrodynamical simulation. These issues will be addressed in future work.

\section{Acknowledgments}

Numerical computations were carried out on the XC30 system at the Center for Computational Astrophysics (CfCA) of the National Astronomical Observatory of Japan. This work was supported in part by Grants-in-Aid for Scientific Research of the Japanese Ministry of Education, Culture, Sports, Science and Technology No.16K17702(YO), No.16J06773(SK), and No.15K05088(RY).

\section{References}

[1] Y. Ohira, K. Murase, and R. Yamazaki, Gamma-Rays from Molecular Clouds Illuminated by Cosmic Rays Escaping from Interacting Supernova Remnants, MNRAS, 410, 1577-1582, 2011

[2] M. Ackermann, et al., Detection of the Characteristic Pion-Decay Signature in Supernova Remnants Science, 339, 807, 2013 
[3] J. R. Jokipii, Rate of energy gain and maximum energy in diffusive shock acceleration, Astrophys. J. 313, 842, 1987

[4] Y. Ohira, Injection to Rapid Diffusive Shock Acceleration at Perpendicular Shocks in Partially Ionized Plasmas, Astrophys. J. 827, 36, 2016

[5] M. Takamoto, and J. G. Kirk, Rapid Cosmic-ray Acceleration at Perpendicular Shocks in Supernova Remnants, Astrophys. J. 809, 29, 2015

[6] Y. Ohira, N. Kawanaka, and K. Ioka, Cosmic-ray hardenings in light of AMS-02 data, PRD 93, 083001,2016

[7] K. P. Watters, and R. W. Romani, The Galactic Population of Young $\gamma$-ray Pulsars, Astrophys. J. 727, 123,2011

[8] S. J. Tanaka, and F. Takahara, A Model of the Spectral Evolution of Pulsar Wind Nebulae, Astrophys. J. 715, 1248, 2010

[9] D. F. Torres, A. Cillis, J. Martin, and E. de Ona Wilhelmi, Time-dependent modeling of TeV-detected, young pulsar wind nebulae, JHEAp, 1, 31, 2014

[10] Y. Ohira, S. Kisaka, and R. Yamazaki, Pulsar Wind Nebulae inside Supernova Remnants as Cosmic-Ray PeVatrons, arXiv:1702.05866, 2017

[11] C. F. McKee, and J. K. Truelove, Explosions in the interstellar medium., Phys. Rep. 256, 157, 1995

[12] E. van der Swaluw, A. Achterberg, Y. A. Gallant, and G. Tóth, Pulsar wind nebulae in supernova remnants. Spherically symmetric hydrodynamical simulations, Astron. Astrophys. 380, 309, 2001

[13] A. R. Bell, Mon. Not. R. Cosmic ray acceleration in pulsar-driven supernova remnants, Astron. Soc. 257, 493, 1992

[14] A. R. Bell, and S. G. Lucek, Cosmic ray acceleration in pulsar-driven supernova remnants: the effect of scattering, Mon. Not. R. Astron. Soc. 283, 1083, 1996

[15] Y. Ohira, K. Murase, and R. Yamazaki, Escape-limited model of cosmic-ray acceleration revisited, Astron. Astrophys. 513, A17 (2010)

[16] Y. Ohira, and K. Ioka, Cosmic-ray Helium Hardening, Astrophys. J. 729, L13 (2011)

[17] Y. Ohira, Simulations of Collisionless Perpendicular Shocks in Partially Ionized Plasmas, PRL, 111, 245002, 2013 